\title{
Scale factor and punch shape effects on the expansion capacities of an aluminum alloy during deep-drawing operations
}

\author{
R. Boissiere ${ }^{1, a}$, P. VACheR ${ }^{2}$ And J.J. Blandin ${ }^{3}$ \\ 1 Institut Jean Lamour, Nancy University, 54000 Nancy, France \\ 2 SYMME, Université de Savoie, 74944 Annecy-Le-Vieux, France \\ 3 SIMAP-GPM2, Institut Polytechnique de Grenoble, 38402 Saint-Martin d'Hères, France
}

Received 9 July 2013, Accepted 21 February 2014

\begin{abstract}
The effects of punch geometry and sample size on forming limit diagrams in expansion are investigated in the case of a 2024 aluminium alloy. Four configurations were selected: flat punch (Marciniak test) or hemispheric punch and decimetric vs. centimetric tooling dimensions. Both decimetric and centimetric deep-drawing devices are associated with an image correlation tool that allows identifying without any contact the deformation on the surface of planar or non-planar specimens. Strains on the surface of the samples are observed by means of a double numerisation in three dimensions of the sample before and after deformation by using stereoscopic vision and triangulation. Finally, deep-drawing limit of the four configurations are compared in expansion state and with literature. Results mainly show that hemispherical punch allows measuring higher strains and is less sensitive to size effect than Marciniak test.
\end{abstract}

Key words: Deep-drawing / forming / aluminium / strain / 3D digitisation

\section{Introduction}

Forming of metal sheet is studied in many technologic fields especially in automotive industry. Determining the forming limit of metallic alloys is then a priority. Several methods can be used to identify such forming limits. The most widely used method is to draw a forming limit diagram from deep drawing tests. In this paper, interest is given to the establishment of such forming limits at room temperature in the case of $2024 \mathrm{Al}$ alloy thanks to deepdrawing tests allowing the measurements of deep-drawing limits in expansion. The forming limit diagram is drawn from the major and minor strains observed at the surface of the sample. This diagram generally corresponds to the boundary between uniform strain and diffuse necking $[1,2]$ but the choice of the limit criteria remains an open question. Data related to Forming Limit Diagram (FLD) of $2024 \mathrm{Al}$ alloy have been reported in the literature (see for instance [3]). Ghosh et al. [4] and Hsu et al. [5] have underlined that the maximal strains are depending on the punch geometry. In the present work, to investigate formability limits of Al 2024 in expansion, major and minor strains data were generated using both Marciniak tests and hemispherical punch tests and both centimetric and decimetric punch dimensions.

\footnotetext{
a Corresponding author:

remi.boissiere@univ-lorraine.fr
}

Moreover, since industry deals increasingly with forming of small components (i.e. mini or micro forming), it is of interest to know if results deduced from a conventional macroscopic deep-drawing device are still suitable for tiny samples formed with a mini forming device? A way to get information about this question is to investigate the influence of dimensions on the strain limit for two devices, decimetric and centimetric.

\section{Experimental devices}

\subsection{Mechanical devices}

Description of macroscopic (decimetric) tooling was detailed elsewhere [6]. The macroscopic sheet metal testing system (decimetric device) is composed of an upper die, a lower blank holder and a punch with a maximal load of $500 \mathrm{kN}$. The clamping force between the die and the blank holder is applied by eight hydraulic jacks and a manual pump. The punch is moving at constant displacement rate $\left(1 \mathrm{~mm} \cdot \mathrm{min}^{-1}\right)$ and automatically stopped when the load drops in order to measure the strain to draw a FLD. In the studied case, failure appears suddenly before the machine stops the test.

At centimetric scale, an original deep-drawing device shown in Figure 1 was developed and adapted on a tensile 

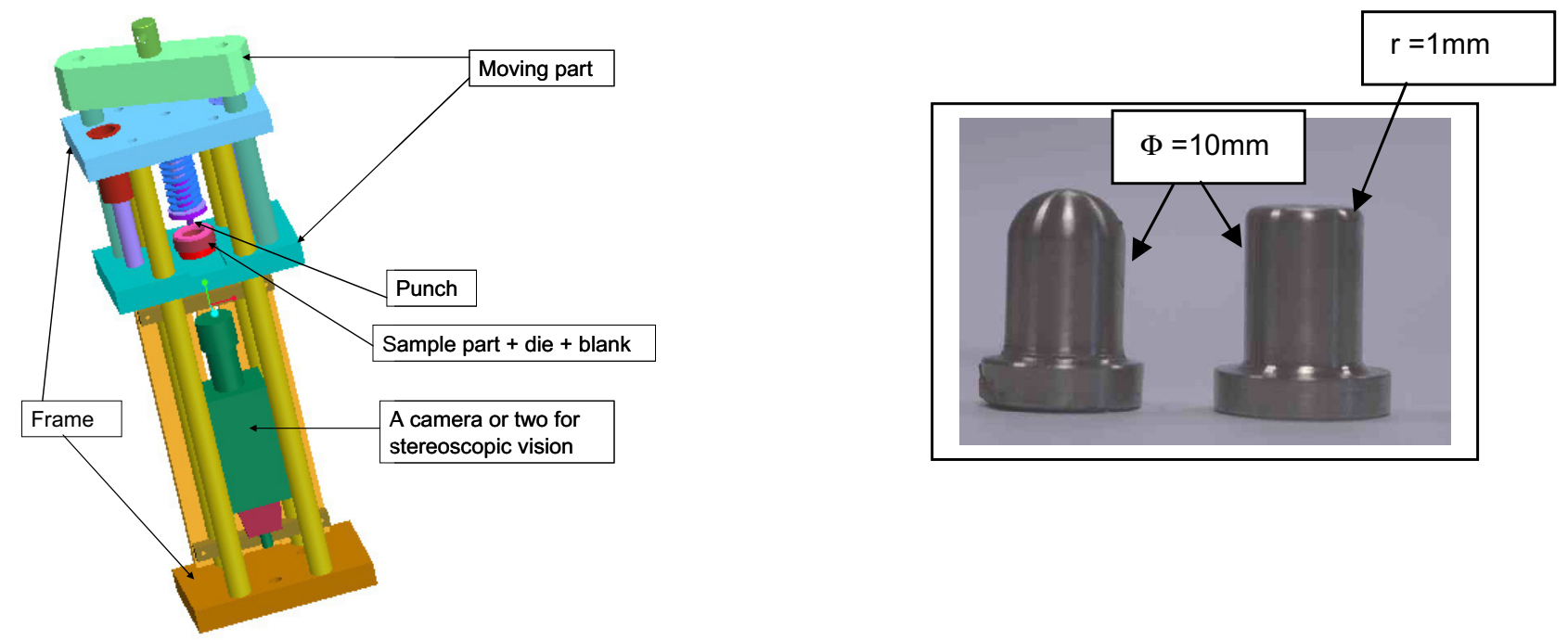

Fig. 1. Scheme of the centimetric device and view of the associated spherical and flat punches.

test machine INSTRON $(50 \mathrm{kN})$. This system allows testing small samples with a punch diameter between $1 \mathrm{~mm}$ and $10 \mathrm{~mm}$.

Experiments from $20^{\circ} \mathrm{C}$ to $250^{\circ} \mathrm{C}$ can be performed thanks to heating sticks inserted into the die. On this device, the punch is fixed; the sample is clamped between the blank holder and the die by the spring supported by a ring guided around the punch. This assembly, linked to the crosshead of the machine by a ball joint, is moved up at a constant velocity of $1 \mathrm{~mm} \cdot \mathrm{min}^{-1}$. This tooling has two interesting features: first, it works in tension allowing better alignment (no buckling effect); secondly it keeps constant the distance between the punch and the fixed camera. This last point is essential if strains are measured by using only one camera (for flat punch applications) but optional for $3 \mathrm{D}$ digitization. The deep-drawing effort is calculated by the difference between the global effort and the spring stiffness, the frictions on the device columns being neglected (Fig. 2).

For both systems, a layer of PTFE is inserted between the specimen and the punch in order to limit frictions. Figure 3 shows the two shapes and dimensions of punches that were used.

The initial thickness of the sheet is $2 \mathrm{~mm}$, value used for decimetric tests. For the centimetric test, the thickness was reduced by using electro-spark cutting techniques. For Marciniak tests, to impose the localisation on the flat surface of the sample, two different methods were undertaken. On the decimetric tool, classical method with a holed spacer between the sample and the PTFE sheet is used. The carrier bank must be centred on the punch in order to provide uniform straining on the flat surface. On the centimetric tool, a new centred local reduction of thickness is obtained again by using an electro-spark cutting process (Fig. 4). This technique permits to get rid of the holed spacer.

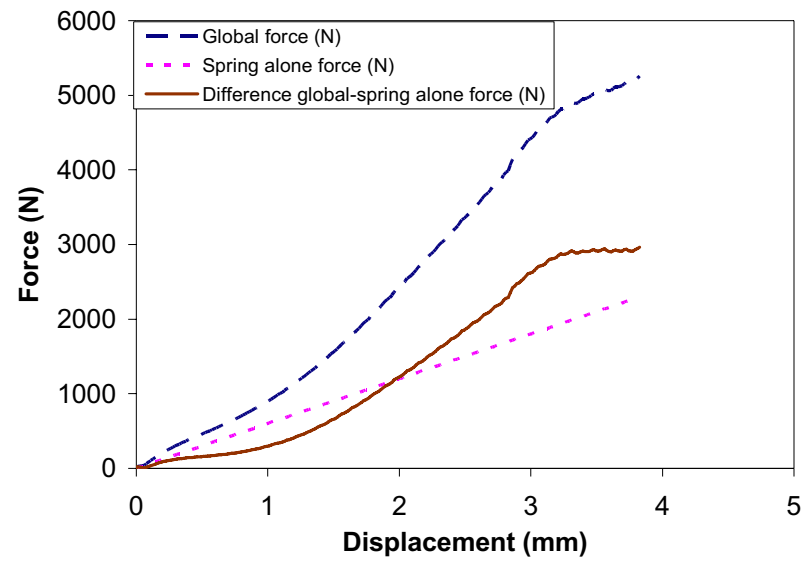

Fig. 2. Variation of load (raw data and corrected taking into account the spring effect) with punch displacement (centimetric device).

\subsection{Strain measurement tools}

Strain fields on the surface were obtained by using images correlation techniques and stereoscopic visions. With these two devices allowing access to bi-axial stretching, it will be shown in the following that various strain situations exist. 3D measurement is adapted to determine the whole range of these strain situations. The chosen comparisons were realised by exploiting these strain fields calculated between the state of the sample before deformation and after the apparition of the first crack. The set of measured points is reported on the strain diagram.

To analyse deformation on a flat surface, a unique camera is enough for a $2 \mathrm{D}$ correlation if this camera is located at a constant distance of the sample, perpendicularly to the flat surface.

Displacement field measurements and calculation of strain fields along non planar surfaces require a double $3 \mathrm{D}$ digitization of this part before and after the 


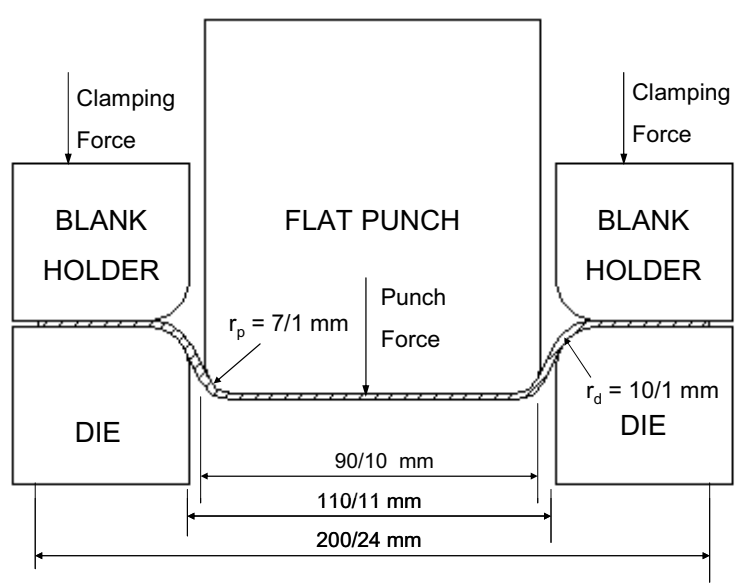

(a)

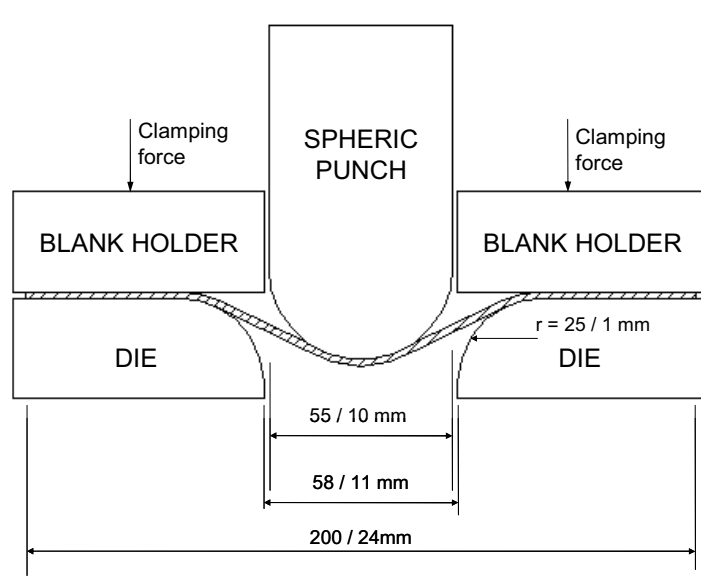

(b)

Fig. 3. (a) Flat punch test (Marciniak test) with dimensions (decimetric punch/centimetric punch). (b) Spherical punch test with dimensions (decimetric punch/centimetric punch).

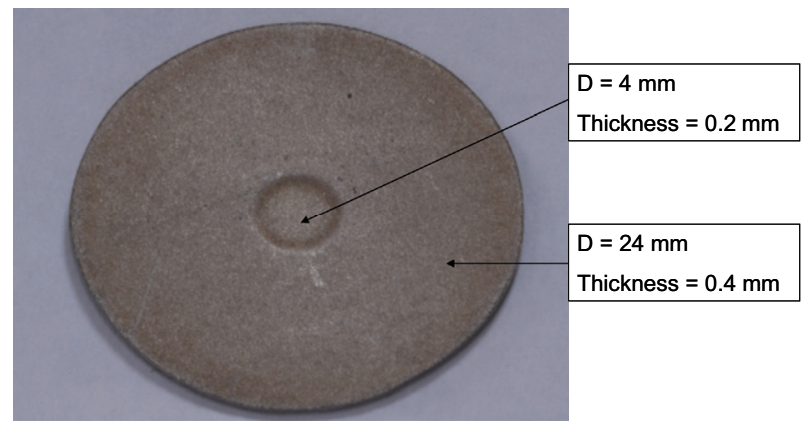

Fig. 4. Observation of the sample with a lower center thickness for Marciniak tests at the centimetric scale.

forming operation. A correlation software "7D" developed by Vacher et al. [7] was used. Acquisition of images was carried out via two synchronised numerical reflex cameras (Nikon D200, $3872 \times 2592$ pixels and macroscopic objective $105 \mathrm{~mm}$ ). Beforehand, the stereoscopic system was calibrated, the relative orientation of the camera was obtained and the optical distortions were corrected [8]. In that case, the angle between the two cameras was close to $15^{\circ}$. The stereoscopic bench is shown in Figure 5 .

In this study, the extensometric dimension (grid step for correlation approach) was chosen close to the thickness of the tested sample: $2 \mathrm{~mm}$ (about 64 pixels) for the decimetric tests with both punches, $0.4 \mathrm{~mm}$ (48 pixels) for the centimetric hemispherical punch and 0.2 (32 pixels) for the centimetric flat punch. The correspondence between the pixel size and the millimetric dimensions was conditioned by the magnification used during the digitization (Table 1).

Defining the displacement measurements uncertainties by this correlation approach is difficult since various parameters can disturb the results such as the maximal strain amplitude, strain gradients, intensity and type of lighting, rigid body rotations, noise of the camera, quality of the random pattern, displacements occurring out of the

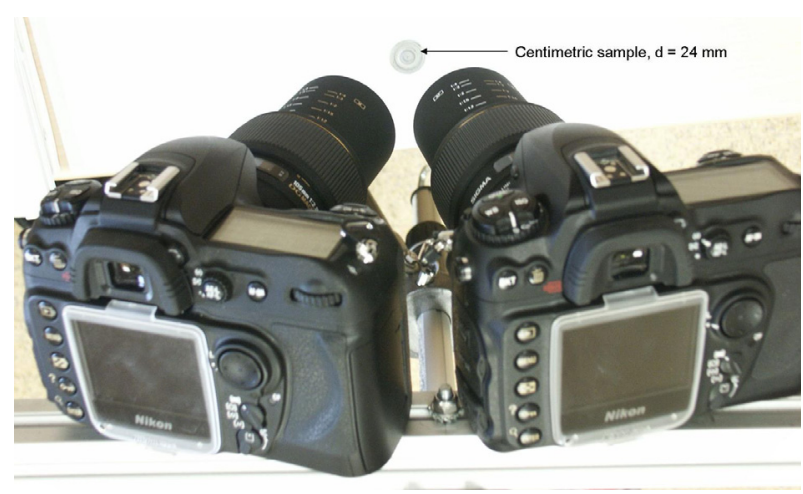

Fig. 5. Stereoscopic bench used for 3D digitization.

planar surfaces. Several authors have shown that these errors in terms of displacement are close to 0.01 pixel for small strains $(<5 \%)$ if these ones are homogeneous [9]. With large strains, these errors are rather close to a tenth of a pixel. In this study, the deformation uncertainties were close to $5 \times 10^{-3}[10]$. In the present investigation, the Green-Lagrange tensor was used with $E_{\min }$ corresponding to the minimal principal strain and $E_{\max }$ related to the maximal principal strain.

\subsection{Material state and characteristics}

The material studied in an aluminium 2024 in the T351 state (thermo-mechanically hardened) that is well known in the aeronautical industry. Its chemical composition is described in Table 2. In this alloy, copper and magnesium are the main alloying elements and are providing a significant raise in the mechanical properties of the alloy.

Concerning the metallurgical state of the specimens, microscopic observations have shown that the microstructure is homogeneous in the thickness of the material 
Table 1. Characteristics of the two systems and extensometric patterns.

\begin{tabular}{lcccc}
\hline & $\begin{array}{c}\text { Punch } \\
\text { diameter } \\
(\mathrm{mm})\end{array}$ & $\begin{array}{c}\text { Thickness } \\
(\mathrm{mm})\end{array}$ & $\begin{array}{c}\text { Extensometric } \\
\text { pattern }(\mathrm{mm})\end{array}$ & $\begin{array}{c}\text { Extensometric } \\
\text { pattern } \\
\text { (pixels) }\end{array}$ \\
\hline $\begin{array}{l}\text { Decimetric spherical } \\
\text { punch (DSP) }\end{array}$ & 55 & 2 & 2 & 64 \\
$\begin{array}{l}\text { Decimetric Flat punch } \\
\text { (DFP) }\end{array}$ & 90 & 2 & 2 & 64 \\
$\begin{array}{l}\text { Small spherical punch } \\
\text { (SSP) }\end{array}$ & 10 & 0.4 & 0.4 & 48 \\
Small flat punch (SFP) & 10 & 0.2 & 0.2 & 32 \\
\hline
\end{tabular}
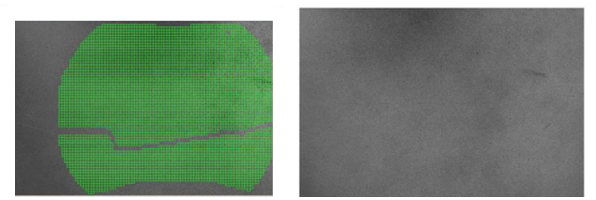

Before deep-drawing
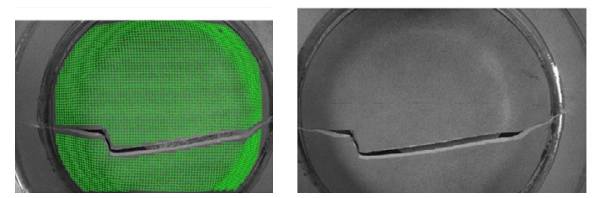

After deep-drawing

Left camera with correlation results, Right camera

Fig. 6. Images before and after deformation of a decimetric Marciniak test sample.

and that no recrystallization phenomena are provoked by electro-spark cutting techniques. The grain size is closed to $20 \mu \mathrm{m}$ and the anisotropy parameter called Lankford coefficient $r$ is closed to two: $r=\varepsilon$ witdh $/ \varepsilon$ thickness $=$ 2. Lankford coefficient is a key factor for deep-drawing capacities. When Lankford coefficient is high $(>2)$, the thickness reduction is lowered and rapid fracture more easily avoided.

\section{Results}

Measurements of 3D fields allow observations of local strains for each configuration. Two stereoscopic images couples were recorded before and after deformation by using a stereoscopic bench (Fig. 6) and the correspondences for each point between the four pictures were obtained by image correlation. Only points gathered from the four images of the specimen can be reconstructed in 3D in the initial and final configurations. Principal strains and their orientations were then calculated. In this paragraph, two tests are described: one using a flat punch with a decimetric size and the other one using a spherical punch with centimetric dimensions.

\subsection{Specificities related to the flat punch tests}

Marciniak test displays the advantage of exhibiting a flat zone that does not undergo any friction with the
Table 2. Chemical composition of Al 2024 alloy.

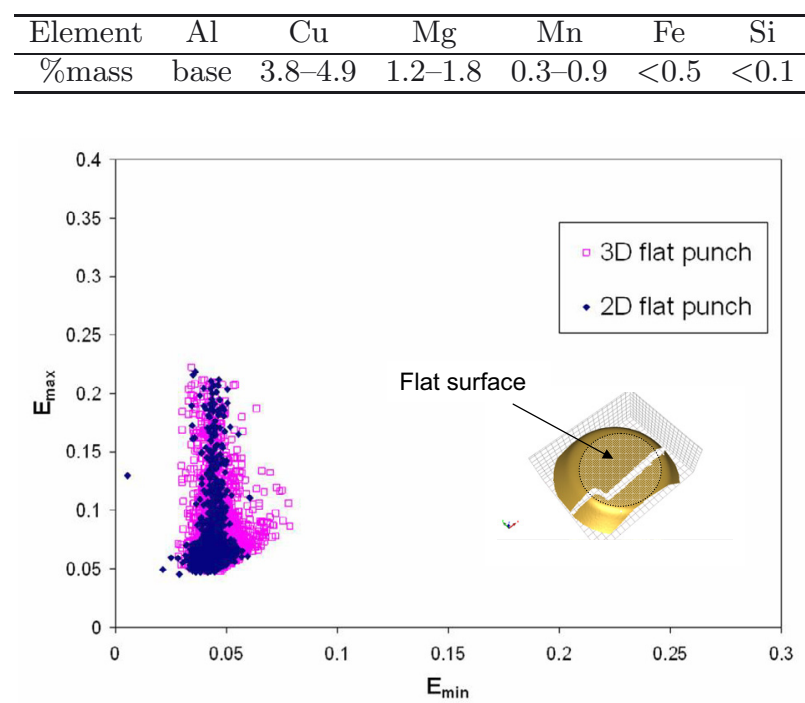

Fig. 7. Emax vs. Emin curves deduced from 2D or 3D analysis in the case of the flat punch for the decimetric tests.

punch. In addition, if necking occurs on the planar surface, the analysis does not require the use of 3D approach. In that case, a 2D correlation with one camera is sufficient as shown in Figure 1. In Figure 7, a comparison of the two types of analysis (2D and $3 \mathrm{D})$ on the same planar surface is presented, the first one using only the data from the camera located perpendicularly to that surface, the second one exploiting the stereoscopic reconstructions. From Figure 7, it can be concluded that the 3D approach leads to similar results than the $2 \mathrm{D}$ one. For all the presented results below, 3D correlation techniques are used, this technique allowing reaching strain situations located on non planar surfaces.

Marciniak tests exhibit nevertheless some drawbacks. Localising the deformation on the planar surface requires the use of a holed spacer or the machining of a local lower sheet thickness, both centred on the punch. Despite that, fracture on the punch entry radius can still occur in particular when using thick sheet or quite brittle alloys. Moreover, for materials displaying anisotropic mechanical behaviours, strain situations are not in pure expansion due to frictions occurring on the edge of the punch which promotes strain along particular directions. 


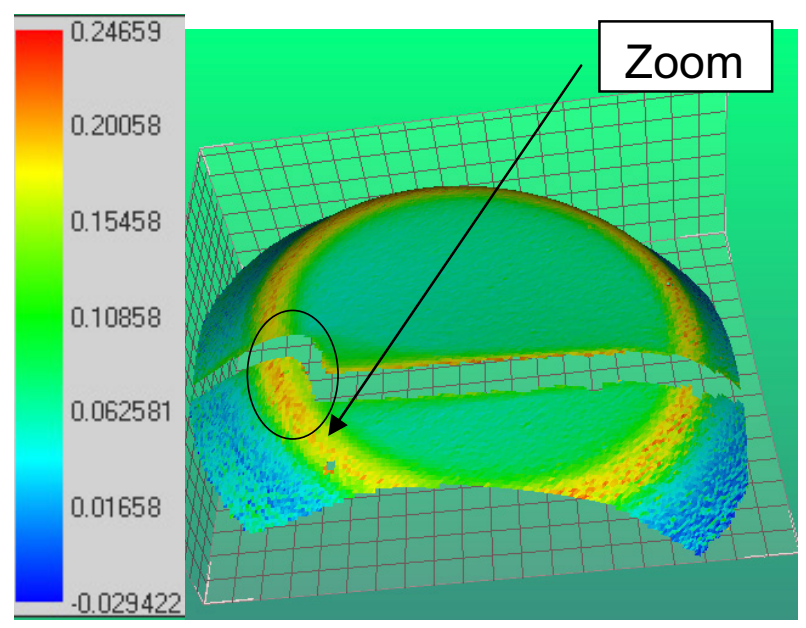

(a)

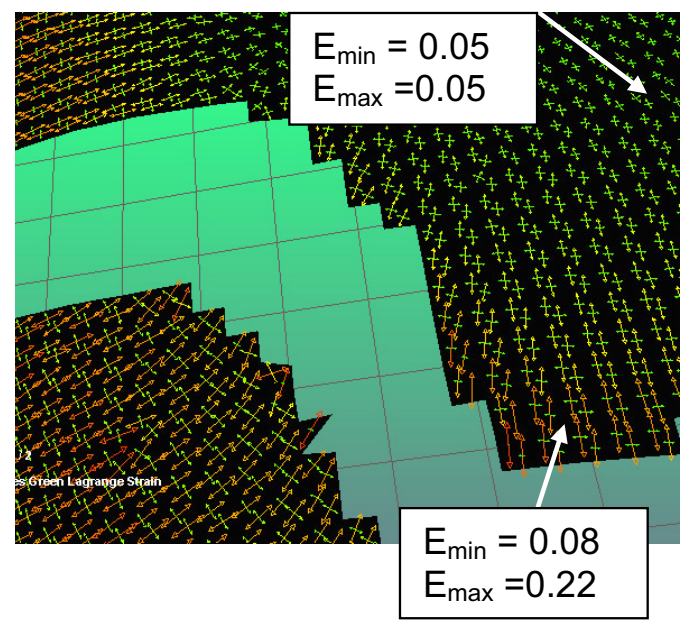

(b)

Fig. 8. (a) Maximal strains after testing with the decimetric flat punch. (b) Deformation state on the zoomed zone: expansion is observed on the area far from the crack with no promoted direction. Note that close to the crack, principal strains are un-balanced.

Figure 8a displays the calculated maximal principal strains on the deformed sample after the deep-drawing test using a decimetric flat punch and Figure $8 \mathrm{~b}$ allows visualizing the principal strains and their directions around the cracked zone. Out of the fractured zone, it can be observed that the higher strains are located on a crown surrounding the flat surface. Moreover, very high strain gradients are located on each side of the crack. Results show that the flat upper zone is in expansion $\left(E_{\min } \approx E_{\max } \approx 0.05\right)$, excepted along the fracture area where the solicitation is close to plane strain $\left(E_{\min } \approx 0.08\right.$ and $E_{\max } \approx 0.22$ ). Curved zones located on the radius of the punch exhibit high strains almost similar to the ones observed along the fracture $\left(E_{\min }=0.07, E_{\max }=0.24\right)$.

Uniaxial tension is occurring in the lateral zones $\left(E_{\min }=-0.06, E_{\max }=0.1\right)$. For the two sizes of flat punch, fracture appeared suddenly. In the case of the centimetric flat punch, fracture is fully localised in the planar zone whereas, for the decimetric punch, the fracture crosses the sample. It is then impossible to know the exact localisation of the fracture first appearance.

Information extracted from these analyses is reported on Figure 9 showing $E_{\min }$ and $E_{\max }$. The two clouds of points correspond to all the calculated points (black points) or only the ones located on the flat zone of the punch (grey points). Usually, analysis of Forming Limit Diagrams (FLD) obtained by using Marcinaik tests is deduced only from the observation of the flat zone. Figure 9 clearly shows that 3D measurements give access to more deformed zones and more various strain distributions in particular on the radius of the punch.

\subsection{Specificities related to the spherical punch tests}

Despite the fact that Marciniak test is a well accepted method for constructing Forming Limit Diagrams, it is

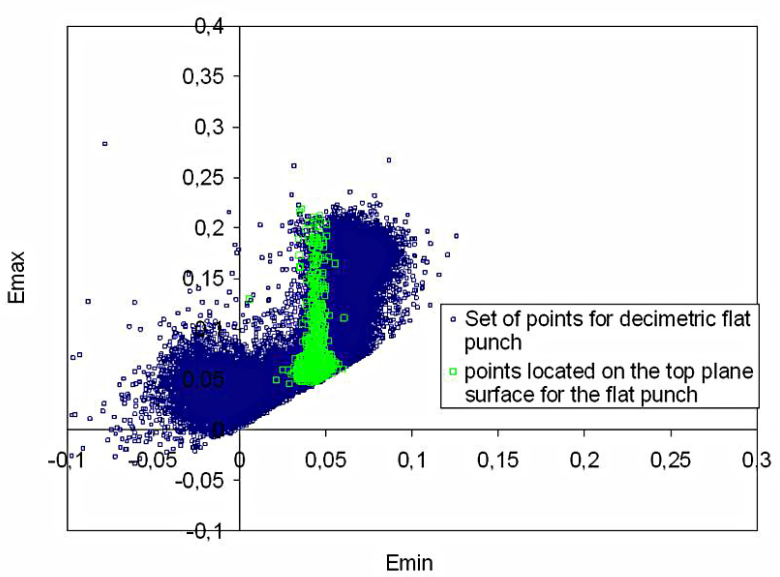

Fig. 9. Emax vs. Emin curves calculated for the decimetric tests using the flat punch.

not the only way to obtain results describing the behaviour of a material in deep-drawing conditions. Hemispheric punch is often used [2], to get the strain limits of a material in expansion. Figures 10 and 11 display information collected in the case of the centimetric device experiments using a spherical punch. From a mechanical point of view, this test is easy to perform since it does not need the use of a holed spacer or a located reduction of the thickness because the strain only concentrates on the spherical zone of the punch. The drawbacks of this method are mainly the influence of bending, normal pressure, and frictions that lead to results which are not very dependent on material defects [4]. During the test, three sample images pairs were recorded: the first one before the test, the second one during the test in which strains are mostly in a bi-axial stretching state, and the last one after fracture. 


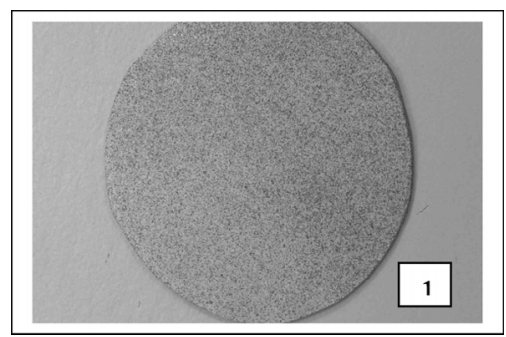

(a)

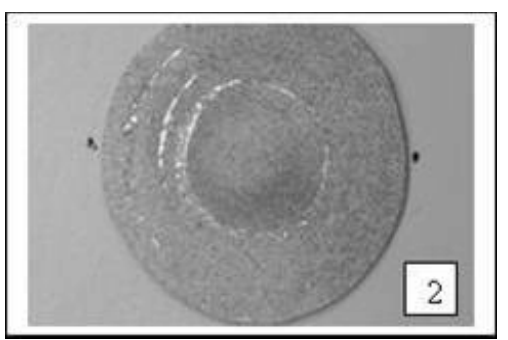

(b)

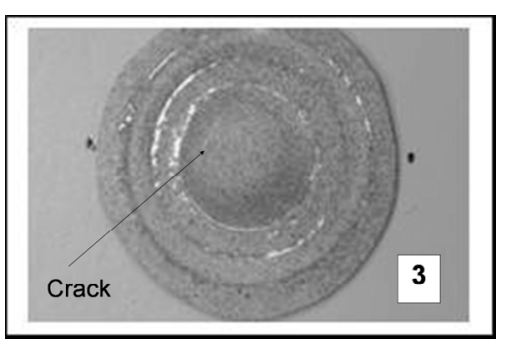

(c)

Fig. 10. (a) Observation of a sample before test (centimetric test). (b) Intermediate strain. (c) Development of the first crack.

It can be seen in Figures 11 a and $11 \mathrm{~b}$ that strains are relatively gathered along an expansion strain situation. As expected, despite the PTFE layer between the punch and the specimen, friction remains significant and reduces the deformation amplitudes on the top part. As a consequence, the central zone is not the most deformed and the maximal strain zone is located within a crown separating the punch contact and the no-contact zone. After fracture, strains in the crack presented in Figure 10b cannot be considered since, in the crack, strains are infinite and the results depend thus hardly on the grid step. In the following, strains calculated in the zones crossing the crack are filtered and the corresponding graphs then only represent the safe zones of each test.

\section{Comparative analysis}

One test supposed in biaxial stretching leads actually to various strain situations and then it is difficult to define only one point summarizing the forming limit of the material. The objective is not to establish an answer in terms of forming limit criteria. Many criteria have been used and the choice of the one that fits best is always hazardous. Criteria concerning the thickness strain can be found, or dealing with the maximal strain observed around the necking zone... [11]. Image correlation techniques have shown that results around the necking are delicate to calculate in order to obtain a proper limit strain. The position of the forming limit curves is strongly dependent on the chosen criterion. In consequence, only the clouds of points will be shown in the following diagrams. The various tests are compared in Figures 12 and 13, regarding the deep-drawing conditions: geometry of the punch (flat or spherical) and scale variations (decimetric or centimetric). The information resulting from the different sizes of flat punches were taken only from the flat surface in order to compare with literature. When using the decimetric tooling, the spherical punch provides a higher strain capacity in the expansion field (Fig. 12a) and allows to reach a maximal strain $E_{\max }=0.35$ with $E_{\min }=0.23$. In the case of the flat punch, one obtains smaller maximal strains $\left(E_{\max } \approx 0.22\right.$ with $E_{\min } \approx 0.1$ ) following a strain distribution between expansion and plane strain. These results are in agreement with the conclusions previously suggested by Ghosh et al. [4]. The observed differences between the two

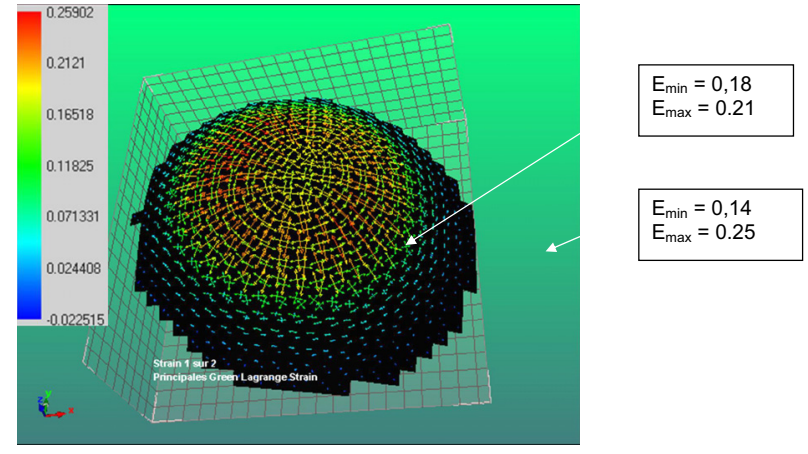

(a)

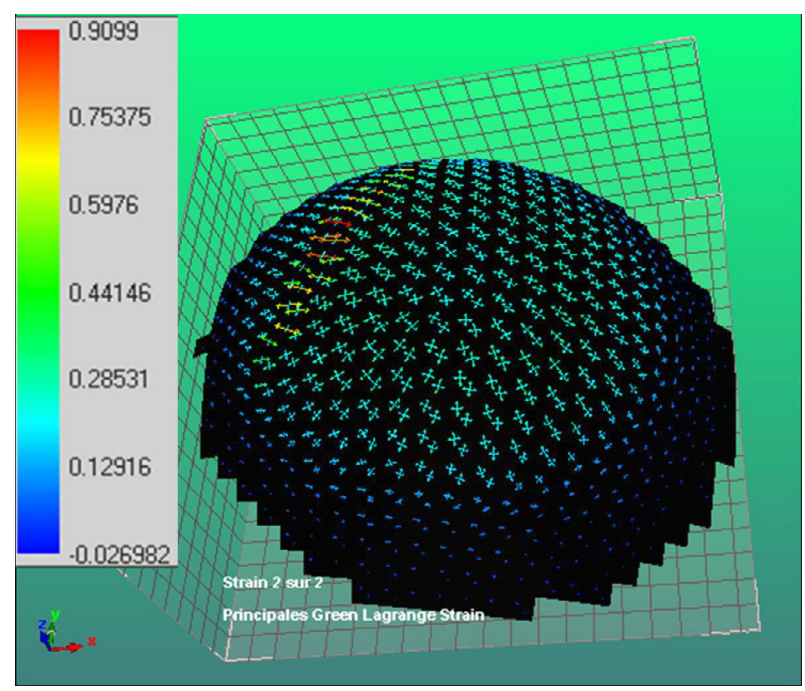

(b)

Fig. 11. (a) Deformation state at intermediate step. Lower strains are already observed on the top of the punch. (b) Deformation map after cracking of the flank.

punches in the decimetric tooling are also relevant for the centimetric device: the spherical punch offers higher strain limits than the flat punch: $E_{\max }=0.25$ (with $E_{\min }=0.23$ which means that $\left.E_{\max } \approx E_{\min }\right)$ with the spherical punch whereas $E_{\max }=0.15$ (with $E_{\min }=0.13$ which means again that $\left.E_{\max } \approx E_{\min }\right)$ with the flat punch. 


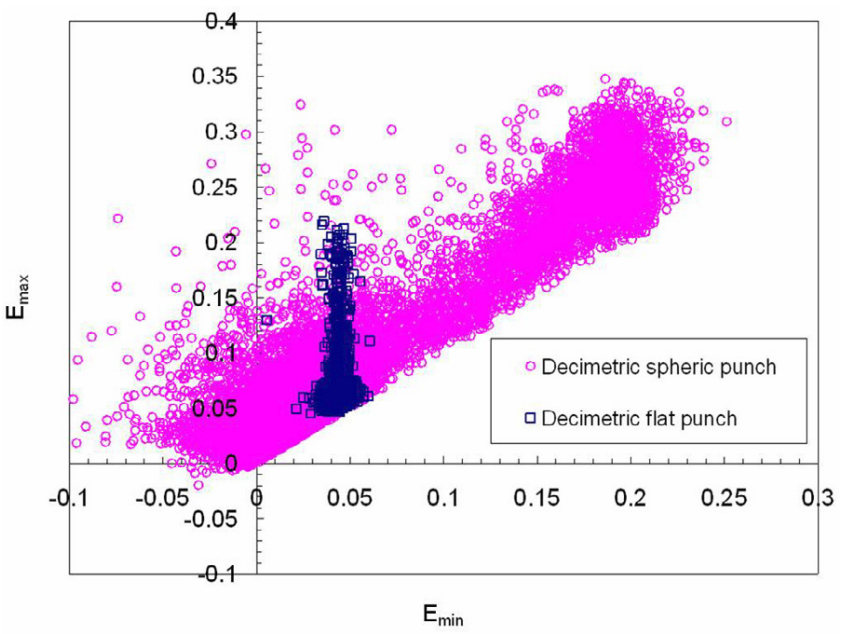

(a)

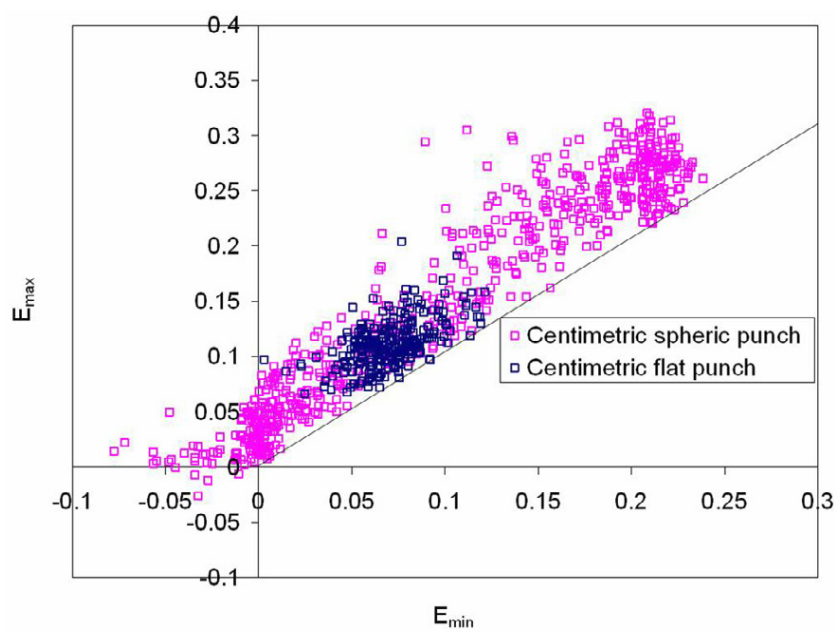

(b)

Fig. 12. (a) Forming diagram in expansion of 2024 alloy using a decimetric device. (b) Forming diagram in expansion using a centimetric device.

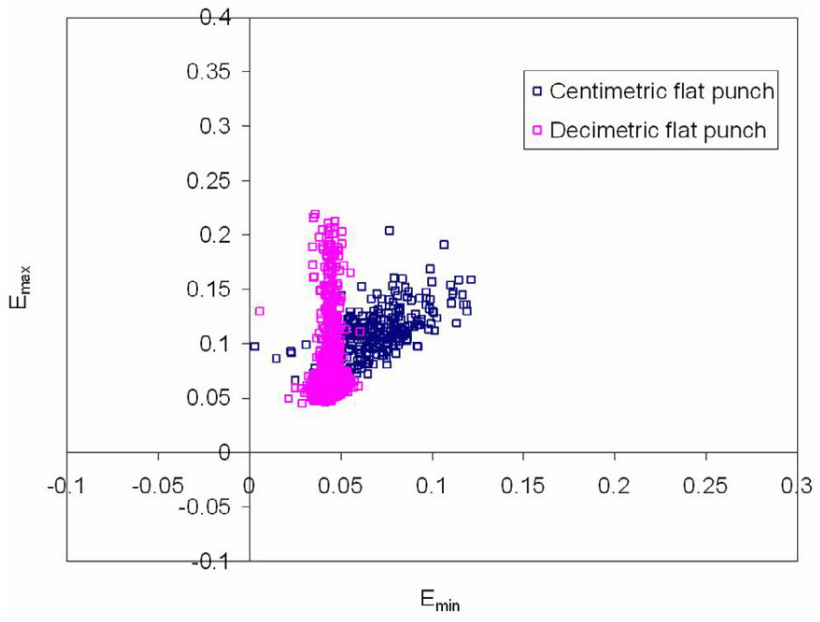

(a)

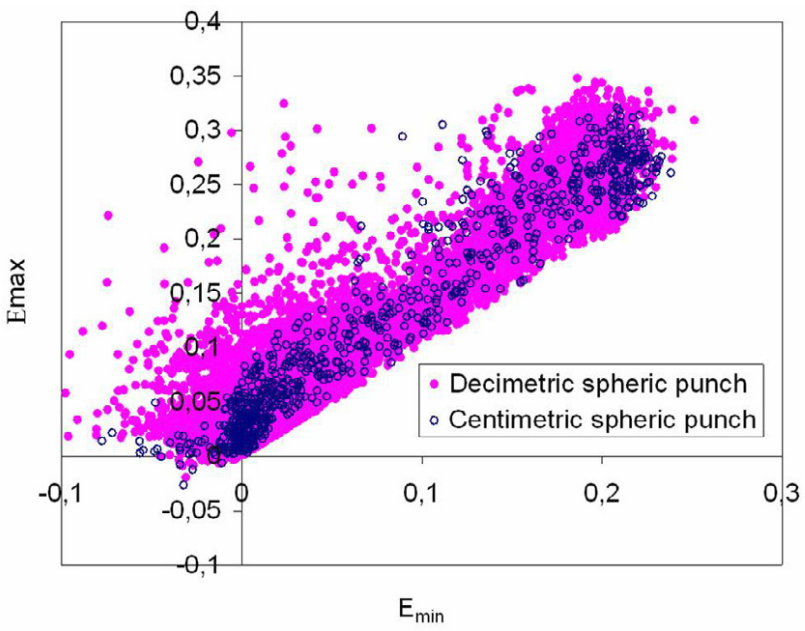

(b)

Fig. 13. (a) Forming diagram in expansion using a flat punch. (b) Forming diagram in expansion using a spherical punch.

It is noteworthy to see that flat punch tests lead to various strain situations depending on the working scale (Fig. 13a). Comparison of the strain distributions on the flat zones is interesting: at decimetric scale, the ratio $E_{\max } / E_{\min }$ between the principal strains close to the crack is about 4 whereas at centimetric scale this ratio remains close to 1.5. At centimetric scale, strong strain heterogeneities are observed. Moreover, defects on the surface or edges of the sample could lead to faster cracking in the case of thinner sheets because the relative sizes of the cracks are more important when the sample is thinner. The spherical centimetric and decimetric tests comparison shows an obvious superposition of the points. It can be observed that the expansion domain is much more extended. To our knowledge, no article is dealing with Al2024 in the T351 state. Reyes et al. [3] have reported some results on a T3 Aluminium 2024. T3 and T351 treatments lead to a similar hardness of the material, one by submitting the material to mechanical hardening and the other by thermo-mechanical treatment. As a result, the observations concerning the conventional decimetric Marciniak test are reliable to compare with the results of the present work: Marciniak homogeneous strains are quite similar (close to 5\%) and most points are located in this uniform zone (Fig. 14).

Some differences can be observed concerning the maximal strains, probably due to the used extensometric pattern. Image correlation allows observing very local strains and then giving access to higher strains in the necking zone of the material.

Nevertheless, spherical punch test offers much higher strains even in the homogeneous zone but for the spherical 

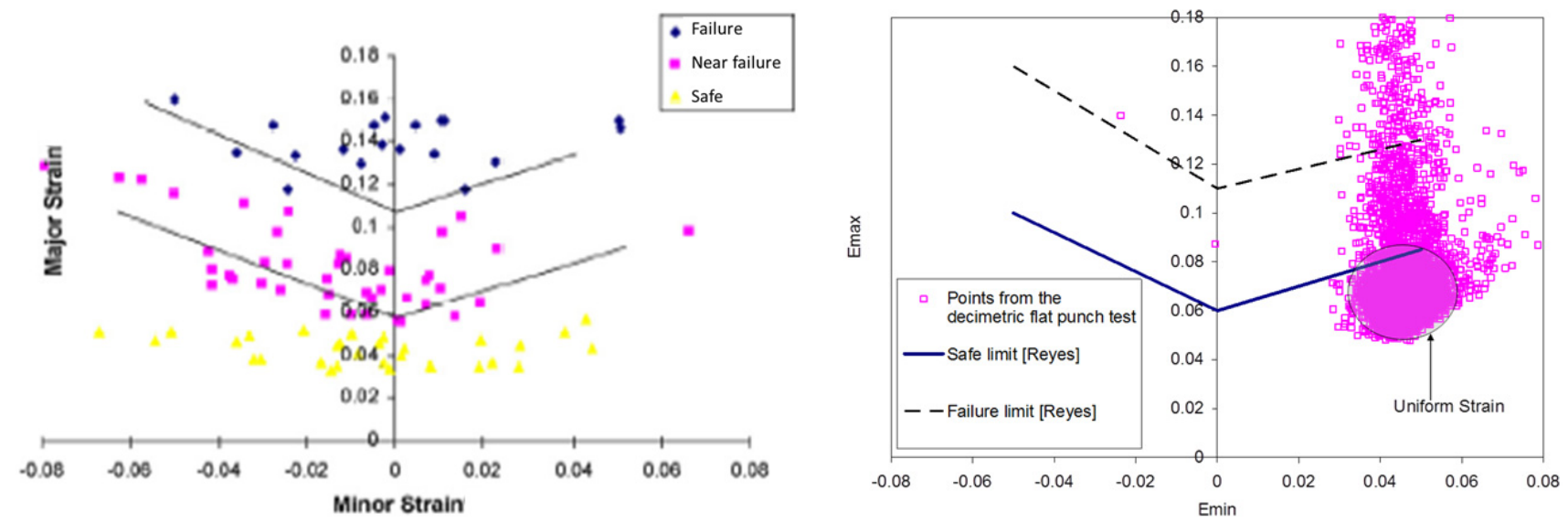

Fig. 14. (a) Formability limit diagram for aluminium alloy 2024-T3 [3], (b) comparison between the present study and [3] results. It can be observed that uniform strain capacities are relatively similar for both works.

punch, no comparison with literature has been found for the studied alloy.

The fact that spherical punch provides higher surface strains can be explained by complementary facts. The surface observed is the external surface solicited in expansion. The use of a spherical punch provides bending with the external surface solicited in multi-axial tension that adds strain to the observed initial expansion. Moreover, friction prevents the internal surface from expanding and then allows the external surface to exhibit higher strains. These points are the main reasons for observing higher strains with the spherical punch compared to the flat one.

Finally, the huge difference between centimetric and decimetric flat punch (Fig. 13) may be due to two main causes: on the centimetric test, defects have more impacts due to their relative size (towards the sample dimensions) which prevent strain to reach a high maximal level ( 0.2 for the decimetric punch). One of the other reasons for disparities is the fact that the holed spacer placed on the decimetric test is not solidar with the sample and then the strain situation is deviated from the expansion one. The differences between the two types of punch are also related to the sample manufacturing. The centimetric one owns a specific shape (lower thickness in the centre of the sample) that is well-controlled whereas the decimetric one needs an external holed spacer for the flat punch that influences the strain distribution. For the use of spherical punches no artefacts are added and so the test is more repetitive when changing the scale of the operation.

\section{Conclusions}

The aim of this study is to investigate the differences between two deep-drawing tests (Marciniak test and hemispheric punch test) and their capacities to be repetitive when changing the tooling and sample dimensions. Results show a significant influence of the punch shape for both device dimensions. Those results are in agreement with the previously reported observations, confirming that larger maximal strains in bi-axial stretching can be obtained with a spherical punch. Concerning the impact of a scale variation, the results obtained with the spherical punch seem to be more reproducible than those obtained with the flat punch. The hemispheric punch is more interesting as it provides various strain situations with only one test. So, with that kind of punch, one can do a limited number of tests at the chosen scale (staying in the range of non-influence of grain size and defect dimensions) in order to obtain a complete forming limit diagram. As a consequence, the use of spherical punch appears as a particularly attractive device to analyse the formability of metals. In future, in order to extend these conclusions to metallic alloys with relatively low ductility, magnesium alloys will be studied and in this framework, an attention will be paid to the effect of temperature on the forming behaviour.

\section{References}

[1] Z. Marciniak, K. Kuczinsky, Int. J.Mech. Sci. 9 (1967) 609-620

[2] R. Arrieux, Ph.D. thesis, INSA and UCB Lyon (F), 1990

[3] G. Reyes, H. Kang, J. Mater. Proc. Tech. 186 (2007) 284290

[4] A.K. Ghosh, S.S. Hecker, Metall. Trans. 5 (1974) 21612164

[5] E. Hsu, J.E. Carsley, R. Verma, J. Mater. Eng. Perf. 17 (2008) 288-296

[6] P. Vacher, S. Dumoulin, R. Arrieux, Intern. J. Forming Proc. 2 (1999) 395-408

[7] P. Vacher, S. Dumoulin, F. Morestin, S. Mguil-Touchal, Proc Instn Mech Engrs, 213 C Imech E, 811-817 (2000)

[8] F. Dumont, Ph.D. thesis, Université Paris 6 - Orléans (F), 2003

[9] M. Bornert, F. Brémand, P. Doumalin, J.C. Dupré, M. Fazzini, M. Grédiac, F. Hild, S. Mistou, J. Molimard, J.J. Orteu, R. Robert, Y. Surrel, P. Vacher, B. Wattrisse, Exp. Mech. (2008)

[10] J.J. Orteu, SEM Annual Conference, Springfield, MA, USA, 2007

[11] P. Vacher, R. Arrieux, L. Tabourot, J. Mater. Proc. Tech. 78 (1998) 190-197

[12] T. Coudert, Ph.D. thesis, Université de Savoie (F), 2005 